\title{
Some fixed point theorems in fuzzy bipolar metric spaces
}

\author{
Ayush Bartwal*, R. C. Dimri, Gopi Prasad \\ Department of Mathematics, HNB Garhwal University, Uttarakhand, India.
}

\begin{abstract}
In this paper, we introduce the notion of fuzzy bipolar metric space and prove some fixed point results in this space. We provide some non-trivial examples to support our claim and also give applications for the usability of the main result in fuzzy bipolar metric spaces.
\end{abstract}

Keywords: Fuzzy metric spaces, fuzzy bipolar metric space, fixed point.

2010 MSC: 54H25, 47H10.

(C)2020 All rights reserved.

\section{Introduction and preliminaries}

In 1960, Schweizer and Sklar [13] introduced the notion of continuous triangular norm. After that in 1965, Zadeh [14] introduces the theory of fuzzy sets. Using the concept of fuzziness, in 1975, Kramosil and Michalek [6] defined the fuzzy metric space with the help of continuous t-norm. The fuzzy approach to the distance follows from the idea that it is not necessary that there always exist a real number to define the distance between any two points which we have to approximate or to find, but it is a fuzzy notion.

In 1994, George and Veeramani [1] modified the definition of fuzzy metric spaces as following.

Definition 1.1 ([1]). Let $Y$ be a nonempty set. An ordered triple $(Y, M, *)$ is called a fuzzy metric space if $\mathrm{M}$ is a fuzzy set on $\mathrm{Y}^{2} \times(0, \infty)$ and $*$ is a continuous t-norm satisfying the following conditions for all $x, y, z \in Y$ and $t, s>0$;

(1) $M(x, y, t)>0$;

(2) $M(x, y, t)=1$ iff $x=y$;

(3) $M(x, y, t)=M(y, x, t)$;

(4) $M(x, z, t+s) \geqslant M(x, y, t) * M(y, z, s)$;

(5) $M(x, y,):.(0, \infty) \longrightarrow(0,1]$ is continuous.

\footnotetext{
${ }^{*}$ Corresponding author

Email addresses: ayushbartwal@gmail.com (Ayush Bartwal), dimrirc@gmail.com (R. C. Dimri), gopiprasad127@gmail.com (Gopi Prasad)
}

doi: $10.22436 /$ jnsa.013.04.04

Received: 2019-07-15 Revised: 2019-08-23 Accepted: 2019-10-31 
Example 1.2. Let $M(x, y, t)=\left[\exp \left(\frac{|x-y|}{t}\right)\right]^{-1} \forall x, y \in \mathbb{R}$ and $t \in(0, \infty)$. Define continuous $t$-norm as $u * v=u v$. Then $(X, \mathbb{R}, *)$ is a fuzzy metric space.

Lemma 1.3 ([8]). Let $(X, M, *)$ be a fuzzy metric space. For all $x, y \in X$ and $t>0$,

$$
M(x, y, k t) \geqslant M(x, y, t),
$$

where $\mathrm{k} \in(0,1)$, then $\mathrm{x}=\mathrm{y}$.

Numerous of generalizations of metric space have been introduced by many eminent mathematicians. Recently, Mutlu and Gurdal [9] generalized metric space which was called bipolar metric spaces and give a new concept of measurement of distance between the elements of two different sets as:

Definition 1.4 ([9]). The 3-tuple $(X, Y, d)$ is said to a bipolar metric space if $X$ and $Y$ are two nonempty sets and $d$ is a function on defined on $X \times Y$ to $\mathbb{R}^{+}$, where $\mathbb{R}^{+}$denotes the set of nonnegative real numbers, satisfying following properties:

(i) if $d(x, y)=0$, then $x=y$ for all $(x, y) \in X \times Y$;

(ii) if $x=y$, then $d(x, y)=0$ for all $(x, y) \in X \times Y$;

(iii) $d(x, y)=d(y, x)$ for all $x, y \in X \cap Y$;

(iv) $d\left(x_{1}, y_{2}\right) \leqslant d\left(x_{1}, y_{1}\right)+d\left(x_{2}, y_{1}\right)+d\left(x_{2}, y_{2}\right)$ for all $x_{1}, x_{2} \in X$ and $y_{1}, y_{2} \in Y$.

Example 1.5. Let $f: \mathbb{R} \rightarrow[1,3]$ and $d: C \times \mathbb{R} \rightarrow \mathbb{R}^{+}$be defined as $d(f, x)=f(x)$. Then $(C, \mathbb{R}, d)$ is a bipolar metric space where $C$ is the set of all functions.

The new notions of generalization and improvement of metric spaces leads to give existence and development of fixed point theorems. As in bipolar metric spaces a lot of significant work has been done by researchers (see, [5, 10-12]) and in fuzzy metric spaces, Grabeic [2] extend the well known fixed point theorem of Banach to fuzzy metric spaces in the sense of Karamosil and Michalek [6]. After that, Gregori and Sapena [3] extended the fuzzy banach contraction theorem to fuzzy metric space in the sense George and Veeramani's [2]. Recently, Gupta et al. [4] proved some applications for fixed point theorems in fuzzy metric spaces.

Definition $1.6([4])$. Define a function $\Theta:[0, \infty) \rightarrow[0, \infty)$, such that $\Theta(t)=\int_{0}^{t} \curlyvee(t) d t, \forall t>0$, be non-decreasing and continuous. Moreover for each $\epsilon>0, \curlyvee(\epsilon)>0$ and $\Upsilon(t)=0$ iff $t=0$.

The aim of this paper is to introduce a new generalization of the fuzzy metric space, which can handle the distance between points of two different sets. We define some basic definitions and extend the Banach contraction theorem for this new generalization. In addition, we also provide examples and applications for the validity of our main results.

\section{Fuzzy bipolar metric spaces}

Definition 2.1. Let $X$ and $Y$ be two non-empty sets. A quadruple $\left(X, Y, M_{b}, *\right)$ is said to be fuzzy bipolar metric space (FB-space), where $*$ is continuous t-norm and $M_{b}$ is a fuzzy set on $X \times Y \times(0, \infty)$, satisfying the following conditions for all $t, s, r>0$ :

(FB-1) $M_{b}(x, y, t)>0$ for all $(x, y) \in X \times Y$;

(FB-2) $M_{b}(x, y, t)=1$ if and only if $x=y$ for $x \in X$ and $y \in Y$;

(FB-3) $M_{b}(x, y, t)=M_{b}(y, x, t)$ for all $x, y \in X \cap Y$;

(FB-4) $M_{b}\left(x_{1}, y_{2}, t+s+r\right) \geqslant M_{b}\left(x_{1}, y_{1}, t\right) * M_{b}\left(x_{2}, y_{1}, s\right) * M_{b}\left(x_{2}, y_{2}, r\right)$ for all $x_{1}, x_{2} \in X$ and $y_{1}, y_{2} \in Y$;

$\left(\right.$ FB-5) $M_{b}(x, y,):.[0, \infty) \rightarrow[0,1]$ is left continuous;

(FB-6) $M_{b}(x, y,$.$) is non-decreasing for all x \in X$ and $y \in Y$. 
Remark 2.2. In fuzzy bipolar metric space $\left(X, Y, M_{b}, *\right), M_{b}(x, y, t)$ can be thought of as the degree of nearness between $x$ and $y$ with respect to $t$, where $(x, y) \in X \times Y$.

Example 2.3. Let $(X, Y, d)$ be an bipolar metric space. For all $x \in X, y \in Y$, and $t>0$, denote

$$
M_{b}(x, y, t)=\frac{t}{t+d(x, y)}
$$

Then $\left(\mathrm{X}, \mathrm{Y}, \mathrm{M}_{\mathrm{b}}, *\right)$ is fuzzy bipolar metric space, where is a $\mathrm{t}$-norm defined as $\mathrm{a} * \mathrm{~b}=\mathrm{ab}$ or $\mathrm{a} * \mathrm{~b}=$ $\min \{a, b\}$.

Remark 2.4. In a fuzzy bipolar metric space $\left(X, Y, M_{b}, *\right)$, if $X=Y$ then $\left(X, M_{b}, *\right)$ is fuzzy metric space.

Definition 2.5. Let $\left(X, Y, M_{b}, *\right)$ be a fuzzy bipolar metric space. The points belong to $X, Y$ and $X \cap Y$ are called as Left, Right and Central Points respectively and sequences belong to $X, Y$ and $X \cap Y$ are named as Left, Right and Central sequences respectively.

Lemma 2.6. Let $\left(\mathrm{X}, \mathrm{Y}, \mathrm{M}_{\mathrm{b}}, *\right)$ be a fuzzy bipolar metric space such that

$$
M_{b}(x, y, k t) \geqslant M_{b}(x, y, t)
$$

for $x \in X, y \in Y$ and $k \in(0,1)$. Then $x=y$.

Proof. We have

$$
M_{b}(x, y, k t) \geqslant M_{b}(x, y, t) \text { for } t>0 .
$$

Since $k t<t$ for all $t>0$ and $k \in(0,1)$, by (FB-6) we have

$$
M_{b}(x, y, k t) \leqslant M_{b}(x, y, t) .
$$

From (2.1) and (2.2) and definition of FB-space we get $x=y$.

Definition 2.7. Let $\left(X, Y, M_{b}, *\right)$ be a fuzzy bipolar metric space. A sequence $\left\{x_{n}\right\} \in X$ converges to a right point $y$ if and only if for every $\epsilon>0$ and $t>0$, there exists $n_{0} \in \mathbb{N}$ such that $M_{b}\left(x_{n}, y, t\right)>1-\epsilon$ for all $n \geqslant n_{0}$, i.e., $M_{b}\left(x_{n}, y, t\right) \rightarrow 1$ as $n \rightarrow \infty$ for all $t>0$ (symbolically $\left\{x_{n}\right\} \rightarrow y$ or $\lim _{n \rightarrow \infty} x_{n}=y$ as $n \rightarrow \infty$ ). Similarly, a right sequence $\left\{y_{n}\right\}$ converges to a left point $x$ if and only if for every $\epsilon>0$ and $t>0$, there exist $n_{0} \in \mathbb{N}$ such that $M_{b}\left(x, y_{n}, t\right)>1-\epsilon$ for all $n \geqslant n_{0}$, i.e., $M_{b}\left(x, y_{n}, t\right) \rightarrow 1$ as $n \rightarrow \infty$ for all $t>0$ (symbolically $\left\{y_{n}\right\} \rightarrow x$ or $\lim _{n \rightarrow \infty} y_{n}=x$ as $n \rightarrow \infty$ ).

Remark 2.8. If $\mathrm{X}=\mathrm{Y}$, then convergent of any sequences in FB-space is equivalent to its convergence in fuzzy metric space.

Definition 2.9. Let $\left(X, Y, M_{b}, *\right)$ be a fuzzy bipolar metric space then:

(i) Sequence $\left(x_{n}, y_{n}\right) \in X \times Y$ is named as a bisequence on $\left(X, Y, M_{b}, *\right)$.

(ii) If both $x_{n}$ and $y_{n}$ converge, the sequence $\left(x_{n}, y_{n}\right) \in X \times Y$ is said to be a convergent sequence. If both $x_{n}$ and $y_{n}$ converge to same center point, bisequence $\left(x_{n}, y_{n}\right)$ is said to be a biconvergent sequence.

(iii) A bisequence $\left(x_{n}, y_{n}\right)$ on FB-space $\left(X, Y, M_{b}, *\right)$ is said to be a Cauchy bisequence if for each $\epsilon>0$, there exists $n_{0} \in \mathbb{N}$ such that for all $n, m \geqslant n_{0}(n, m \in \mathbb{N})$, we have $M_{b}\left(x_{n}, y_{m}, t\right)>1-\epsilon$ for each $t>0$, i.e., a bisequence $\left(x_{n}, y_{n}\right)$ is said to be a Cauchy bisequence if $M_{b}\left(x_{n}, y_{m}, t\right) \rightarrow 1$ as $n, m \rightarrow \infty$ for all $t>0$.

Definition 2.10. The fuzzy bipolar metric space $\left(X, Y, M_{b}, *\right)$ is said to be complete if every Cauchy bisequence in $\mathrm{X} \times \mathrm{Y}$ is convergent in it. 
Proposition 2.11. In a fuzzy bipolar metric space, every convergent Cauchy bisequence is biconvergent.

Proof. Let $\left(X, Y, M_{b}, *\right)$ be a fuzzy bipolar metric space and a bisequence $\left(x_{n}, y_{n}\right) \in X \times Y$ such that $\left\{x_{n}\right\} \rightarrow y \in Y$ and $\left\{y_{n}\right\} \rightarrow x \in X$. Since $\left(x_{n}, y_{n}\right)$ is convergent Cauchy bisequence, so for all $t>0$ we have

$$
M_{b}\left(x_{n}, y_{n}, t\right) \rightarrow 1 \text { as } n \rightarrow \infty,
$$

which implies that

$$
M_{b}(x, y, t)=1 \text { for all } t>0 .
$$

Hence by (FB-2) bisequence $\left(x_{n}, y_{n}\right)$ is biconvergent.

Proposition 2.12. In a fuzzy bipolar metric space, every biconvergent bisequence is a Cauchy bisequence.

Proof. Let $\left(X, Y, M_{b}, *\right)$ be a fuzzy bipolar metric space and bisequence $\left(x_{n}, y_{n}\right) \in X \times Y$ converges to a point $x_{0} \in X \cap Y$ for all $n, m \in \mathbb{N}$ and $t>0$, by FB- 4 we have

$$
M_{b}\left(x_{n}, y_{m}, t\right) \geqslant M_{b}\left(x_{n}, x_{0}, \frac{t}{3}\right) * M_{b}\left(x_{0}, x_{0}, \frac{t}{3}\right) * M_{b}\left(u, y_{m}, \frac{t}{3}\right)
$$

as $n, m \rightarrow \infty$, we get

$$
M_{b}\left(x_{n}, y_{m}, t\right) \geqslant 1 \text { for all } t>0 .
$$

Which implies that $M_{b}\left(x_{n}, y_{m}, t\right) \rightarrow 1$ for all $t>0$. Hence, $\left(x_{n}, y_{n}\right)$ is a Cauchy bisequence.

Lemma 2.13. Let $\left(\mathrm{X}, \mathrm{Y}, \mathrm{M}_{\mathrm{b}}, *\right)$ be a fuzzy bipolar metric space and $\mathrm{u} \in \mathrm{X} \cap \mathrm{Y}$ is a limit of a sequence then it is a unique limit of the sequence.

Proof. Let $\left\{x_{n}\right\} \in X$ be a sequence. Suppose that $x_{n} \rightarrow y \in Y$ and also $\left\{x_{n}\right\} \rightarrow u \in X \cap Y$, then for $t, s, r>0$ we have

$$
M_{b}(u, y, t+s+r) \geqslant M_{b}(u, u, t) * M_{b}\left(x_{n}, u, s\right) * M_{b}\left(x_{n}, y, r\right)
$$

as $n \rightarrow \infty$ we get

$$
M_{b}(u, y, t+s+r) \geqslant 1,
$$

which implies that $u=y$, i.e., sequence $\left\{x_{n}\right\}$ have a unique limit.

\section{Main results}

In this section, we prove the extension of some well known fixed point theorems to fuzzy bipolar metric spaces.

Theorem 3.1. Let $\left(\mathrm{X}, \mathrm{Y}, \mathrm{M}_{\mathrm{b}}, *\right)$ be a complete fuzzy bipolar metric space such that

$$
\lim _{t \rightarrow \infty} M_{b}(x, y, t)=1 \text { for all } x \in X, y \in Y
$$

Let $\mathrm{T}: \mathrm{X} \cup \mathrm{Y} \rightarrow \mathrm{X} \cup \mathrm{Y}$ be mapping satisfying

(i) $\mathrm{T}(\mathrm{X}) \subseteq \mathrm{X}$ and $\mathrm{T}(\mathrm{Y}) \subseteq \mathrm{Y}$;

(ii) $M_{b}(T(x), T(y), k t) \geqslant M_{b}(x, y, t)$ for all $x \in X, y \in Y$ and $t>0$, where $k \in(0,1)$.

Then $\mathrm{T}$ has a unique fixed point.

Proof. Fix $x_{0} \in X$ and $y_{0} \in Y$ and assume that $T\left(x_{n}\right)=x_{n+1}$ and $T\left(y_{n}\right)=y_{n+1}$ for all $n \in \mathbb{N} \cup\{0\}$. Then we get $\left(x_{n}, y_{n}\right)$ as a bisequence on fuzzy bipolar metric space $\left(X, Y, M_{b}, *\right)$. Now, we have

$$
M_{b}\left(x_{1}, y_{1}, t\right)=M_{b}\left(T\left(x_{0}\right), T\left(y_{0}\right), t\right) \geqslant M_{b}\left(x_{0}, y_{0}, \frac{t}{k}\right)
$$

for all $t>0$ and $n \in \mathbb{N}$. By simple induction we get 


$$
M_{b}\left(x_{n}, y_{n}, t\right)=M_{b}\left(T\left(x_{n-1}\right), T\left(y_{n-1}\right), t\right) \geqslant M_{b}\left(x_{0}, y_{0}, \frac{t}{k^{n}}\right)
$$

and

$$
M_{b}\left(x_{n+1}, y_{n}, t\right)=M_{b}\left(T\left(x_{n}\right), T\left(y_{n-1}\right), t\right) \geqslant M_{b}\left(x_{1}, y_{0}, \frac{t}{k^{n}}\right)
$$

for all $t>0$ and $n \in \mathbb{N}$.

Letting $n<m$, for $n, m \in \mathbb{N}$; if we take $g_{i} \in(0,1)$ for $i=n, \ldots, 2(m-n)+1$, satisfying $g_{n}+\cdots+$ $g_{2(m-n)+1}$. Then from the definition of FB-space, (3.2) and (3.3), we get

$$
\begin{aligned}
M_{b}\left(x_{n}, y_{m}, t\right) \geqslant & M_{b}\left(x_{n}, y_{n}, g_{n} t\right) * M_{b}\left(x_{n+1}, y_{n}, g_{n+1} t\right) * \cdots \\
& * M_{b}\left(x_{m}, y_{m-1}, g_{2(m-n)} t\right) * M_{b}\left(x_{m}, y_{m}, g_{2(m-n)+1} t\right) \\
\geqslant & M_{b}\left(x_{0}, y_{0}, \frac{g_{n} t}{k^{n}}\right) * M_{b}\left(x_{1}, y_{0}, \frac{g_{n+1} t}{k^{n}}\right) * \cdots \\
& * M_{b}\left(x_{1}, y_{0}, \frac{g_{2(m-n) t}}{k^{m-1}}\right) * M_{b}\left(x_{0}, y_{0}, \frac{g_{2(m-n)+1} t}{k^{m}}\right) .
\end{aligned}
$$

From (3.1), as $n, m \rightarrow \infty$ we get

$$
M_{b}\left(x_{n}, y_{m}, t\right) \geqslant 1 \text { for all } t>0 .
$$

Which implies that bisequence $\left(x_{n}, y_{n}\right)$ is a Cauchy bisequence. Now, FB-space $\left(X, Y, M_{b}, *\right)$ is a complete space. So, bisequence $\left(x_{n}, y_{n}\right)$ is a convergent Cauchy bisequence. According to the Proposition 2.11 the bisequence $\left(x_{n}, y_{n}\right)$ is biconvergent sequence.

As, bisequence $\left(x_{n}, y_{n}\right)$ is biconvergent then there exist a point $u \in X \cap Y$ which is a limit of the both sequences $\left\{x_{n}\right\}$ and $\left\{y_{n}\right\}$. By Lemma 2.13, both sequences $\left\{x_{n}\right\}$ and $\left\{y_{n}\right\}$ has a unique limit.

Let we claim that $u \in X \cap Y$ is a fixed point of T. From FB-4, consider

$$
M_{b}(T(u), u, t) \geqslant M_{b}\left(T(u), T\left(y_{n}\right), \frac{t}{3}\right) * M_{b}\left(T\left(x_{n}\right), T\left(y_{n}\right), \frac{t}{3}\right) * M_{b}\left(T\left(x_{n}\right), u, \frac{t}{3}\right)
$$

for all $n \in \mathbb{N}$ and $t>0$ and as $n \rightarrow \infty$ we have

$$
\mathrm{M}_{\mathrm{b}}(\mathrm{T}(\mathrm{u}), \mathrm{u}, \mathrm{t}) \rightarrow 1 * 1 * 1=1 .
$$

From FB-2 we get $\mathrm{T}(\mathrm{u})=u$.

For the uniqueness of the fixed point assume that a point $v \in X \cap Y$ is another fixed point of T. We have

$$
M_{b}(u, v, t)=M_{b}(T(u), T(v), t) \geqslant M_{b}\left(u, v, \frac{t}{k}\right)
$$

for $k \in(0,1)$ and for all $t>0$. By Lemma 2.6 we get $u=v$.

Example 3.2. Let $X=[0,1]$ and $Y=\{0\} \cup \mathbb{N}-\{1\}$. Define $M_{b}=\frac{t}{t+|x-y|}$ for all $t>0$ and $x \in X$ and $y \in Y$. Clearly, $\left(X, Y, M_{b}, *\right)$ is a complete fuzzy bipolar metric space, where $*$ is a continuous t-norm defined as $a * b=a b$.

Let $T: X \cup Y \rightarrow X \cup Y$ be a mapping given by

$$
T(u)= \begin{cases}\frac{u}{2}, & \text { if } u \in[0,1], \\ 0, & \text { if } u \in \mathbb{N}-\{1\},\end{cases}
$$

for all $u \in X \cup Y$. T satisfies the condition (i) of Theorem 3.1. Now, suppose that $k=\frac{1}{2}$ then for all $t>0$, condition (ii) of Theorem 3.1 also satisfies by T. We can construct the bisequences $T\left(x_{n}\right)=x_{n+1}$ and $T\left(y_{n}\right)=y_{n+1}$ for all $n \in \mathbb{N} \cup\{0\}$ by assuming $x_{0}=1$ and $y_{0}=2$, we obtain a non-trivial sequence as $\left(x_{n}, y_{n}\right)=\left\{(1,2),\left(\frac{1}{2}, 0\right),\left(\frac{1}{2^{2}}, 0\right), \ldots\right\}$. By Theorem 3.1 , we get $\mathrm{T}$ have a unique fixed point, i.e., $x=0$. 
Corollary 3.3. Let $\left(\mathrm{X}, \mathrm{Y}, \mathrm{M}_{\mathrm{b}}, *\right)$ be a complete fuzzy bipolar metric space such that

$$
\lim _{t \rightarrow \infty} M_{b}(x, y, t)=1 \text { for all } x \in X, y \in Y .
$$

Let $\mathrm{T}: \mathrm{X} \cup \mathrm{Y} \rightarrow \mathrm{X} \cup \mathrm{Y}$ be a mapping satisfying

(i) $\mathrm{T}(\mathrm{X}) \subseteq \mathrm{Y}$ and $\mathrm{T}(\mathrm{Y}) \subseteq \mathrm{X}$;

(ii) $M_{b}(T(y), T(x), k t) \geqslant M_{b}(x, y, t)$ for all $x \in X, y \in Y$ and $t>0$, where $k \in(0,1)$.

Then $T$ has a unique fixed point.

Proof. Fix $x_{0} \in X$ and assume that $T\left(x_{n}\right)=y_{n}$ and $T\left(y_{n}\right)=x_{n+1}$ for all $n \in \mathbb{N} \cup\{0\}$. Then we get $\left(x_{n}, y_{n}\right)$ as a bisequence on fuzzy bipolar metric space $\left(X, Y, M_{b}, *\right)$. Now, we have

$$
M_{b}\left(x_{1}, y_{0}, t\right)=M_{b}\left(T\left(y_{0}\right), T\left(x_{0}\right), t\right) \geqslant M_{b}\left(x_{0}, y_{0}, \frac{t}{k}\right)
$$

for all $t>0$ and $n \in \mathbb{N}$. By simple induction we get

$$
M_{b}\left(x_{n}, y_{n}, t\right)=M_{b}\left(T\left(y_{n-1}\right), T\left(x_{n}\right), t\right) \geqslant M_{b}\left(x_{0}, y_{0}, \frac{t}{k^{2 n}}\right)
$$

and

$$
M_{b}\left(x_{n+1}, y_{n}, t\right)=M_{b}\left(T\left(y_{n}\right), T\left(x_{n}\right), t\right) \geqslant M_{b}\left(x_{0}, y_{0}, \frac{t}{k^{2 n+1}}\right)
$$

for all $t>0$ and $n \in \mathbb{N}$.

Letting $n<m$, for $n, m \in \mathbb{N}$; if we take $g_{i} \in(0,1)$ for $i=n, \ldots, 2(m-n)+1$, satisfying $g_{n}+\cdots+$ $g_{2(m-n)+1}$, then from the definition of FB-space, (3.5) and (3.6), we get

$$
\begin{aligned}
M_{b}\left(x_{n}, y_{m}, t\right) \geqslant & M_{b}\left(x_{n}, y_{n}, g_{n} t\right) * M_{b}\left(x_{n+1}, y_{n}, g_{n+1} t\right) * \cdots \\
& * M_{b}\left(x_{m}, y_{m-1}, g_{2(m-n)} t\right) * M_{b}\left(x_{m}, y_{m}, g_{2(m-n)+1} t\right) \\
\geqslant & M_{b}\left(x_{0}, y_{0}, \frac{g_{n} t}{k^{2 n}}\right) * M_{b}\left(x_{0}, y_{0}, \frac{g_{n+1} t}{k^{2 n+1}}\right) * \cdots \\
& * M_{b}\left(x_{0}, y_{0}, \frac{g_{2(m-n) t}}{k^{2 m+1}}\right) * M_{b}\left(x_{0}, y_{0}, \frac{g_{2(m-n)+1} t}{k^{2 m}}\right) .
\end{aligned}
$$

From (3.4), as $n, m \rightarrow \infty$ we get

$$
M_{b}\left(x_{n}, y_{m}, t\right) \geqslant 1 \text { for all } t>0 .
$$

Which implies that bisequence $\left(x_{n}, y_{n}\right)$ is a Cauchy bisequence. Now, FB-space $\left(X, Y, M_{b}, *\right)$ is a complete space. So, bisequence $\left(x_{n}, y_{n}\right)$ is a convergent Cauchy bisequence. According to the Proposition 2.11 the bisequence $\left(x_{n}, y_{n}\right)$ is biconvergent sequence.

As, bisequence $\left(x_{n}, y_{n}\right)$ is biconvergent then there exist a point $u \in X \cap Y$ which is a limit of the both sequences $\left\{x_{n}\right\}$ and $\left\{y_{n}\right\}$. By, Lemma 2.13 both sequences $\left\{x_{n}\right\}$ and $\left\{y_{n}\right\}$ have a unique limit. Now, to prove $u \in X \cap Y$ as the fixed point of mapping $T$, Let we consider

$$
M_{b}(T(u), u, t) \geqslant M_{b}\left(T(u), T\left(x_{n}\right), \frac{t}{3}\right) * M_{b}\left(T\left(y_{n}\right), T\left(x_{n}\right), \frac{t}{3}\right) * M_{b}\left(u, T\left(x_{n}\right), \frac{t}{3}\right)
$$

for all $n \in \mathbb{N}$ and $t>0$ and as $n \rightarrow \infty$ we have

$$
\mathrm{M}_{\mathrm{b}}(\mathrm{T}(\mathrm{u}), \mathrm{u}, \mathrm{t}) \rightarrow 1 * 1 * 1=1 .
$$

from FB-2 we get $\mathrm{T}(\mathrm{u})=u$.

For the uniqueness of the fixed point assume that a point $v \in X \cap Y$ is another fixed point of T. We have

$$
M_{b}(u, v, t)=M_{b}(T(v), T(u), t) \geqslant M_{b}\left(u, v, \frac{t}{k}\right)
$$

for $k \in(0,1)$ and for all $t>0$. By Lemma 2.6 we get $u=v$. 
Example 3.4. Let $X=\{0,1,2,7\}$ and $Y=\left\{0, \frac{1}{4}, \frac{1}{2}, 3\right\}$ and define a continuous t-norm as $r * s=\min \{r, s\}$. Now, define $M_{b}(x, y, t)=\exp ^{-\frac{|x-y|}{t}}$ for all $t>0, x \in X$ and $y \in Y$. Then $\left(X, Y, M_{b}, *\right)$ is a complete fuzzy bipolar metric space. Suppose we define a mapping $T: X \cup Y \rightarrow X \cup Y$ such that

$$
T(u)= \begin{cases}\frac{1}{4}, & \text { if } u \in\{7,2\}, \\ 0, & \text { if } u \in\left\{0, \frac{1}{4}, \frac{1}{2}, 1,3\right\} .\end{cases}
$$

$\mathrm{T}$ satisfies condition (i) of Corollary 3.3. Fix $x_{0}=7$ and assume $T\left(x_{n}\right)=y_{n}$ and $T\left(y_{n}\right)=x_{n+1}$ for all $n \in \mathbb{N} \cup\{0\}$, we get a non-trivial bisequence $\left(x_{n}, y_{n}\right)=\left\{\left(7, \frac{1}{4}\right),(0,0), \ldots\right\}$. Letting $k=\frac{1}{2}$ we can easily get condition (ii) of Corollary 3.3. Now, according to the hypotheses of Corollary 3.3, $\mathrm{T}$ has a unique fixed point, that is $x=0$.

For weak B-contraction, Mihet [7] defined an increasing function $\Psi:(0,1] \rightarrow(0,1]$ such that $\lim _{n \rightarrow \infty} \Psi^{n}(\kappa)=$ 1 and $\Psi(\kappa) \geqslant \kappa$ for all $\kappa \in(0,1]$. We are proving following fixed point theorem in Bipolar fuzzy metric spaces by using weak B-contraction.

Theorem 3.5. Let $\left(\mathrm{X}, \mathrm{Y}, \mathrm{M}_{\mathrm{b}}, *\right)$ be a complete fuzzy bipolar metric space and $\mathrm{T}: \mathrm{X} \cup \mathrm{Y} \rightarrow \mathrm{X} \cup \mathrm{Y}$ a mapping satisfying:

(i) $\mathrm{T}(\mathrm{X}) \subseteq \mathrm{X}$ and $\mathrm{T}(\mathrm{Y}) \subseteq \mathrm{Y}$;

(ii) For $x \in X, y \in Y$ and $t>0, M_{b}(x, y, t)>0 \Longrightarrow M_{b}(T(x), T(y), t) \geqslant \Psi\left(M_{b}(x, y, t)\right)$.

Then $\mathrm{T}$ has a fixed point.

Proof. Let $x_{0} \in X$ and $y_{0} \in Y$ be such that $T\left(x_{n}\right)=x_{n+1}$ and $T\left(y_{n}\right)=y_{n+1}$ for all $n \in \mathbb{N} \cup\{0\}$, then $\left(x_{n}, y_{n}\right)$ be a bisequence on fuzzy bipolar metric space $\left(X, Y, M_{b}, *\right)$. By the definition of (FB-1) for all $t>0$ and condition (ii) we have

$$
M_{b}\left(x_{n}, y_{n}, t\right) \geqslant \Psi^{n}\left(M_{b}\left(x_{0}, y_{0}, t\right)\right)
$$

and

$$
M_{b}\left(x_{n+1}, y_{n}, t\right) \geqslant \Psi^{n}\left(M_{b}\left(x_{1}, y_{0}, t\right)\right) .
$$

Letting $n<m$, and defining $g_{i}$ same as Theorem 3.1 for all $i=n, \ldots, 2(m-n)+1$, then from (3.7), (3.8), and definition of FB-space we have

$$
\begin{aligned}
M_{b}\left(x_{n}, y_{m}, t\right) \geqslant & \Psi^{n}\left(M_{b}\left(x_{0}, y_{0}, g_{n} t\right)\right) * \Psi^{n} M_{b}\left(\left(x_{1}, y_{0}, g_{n+1} t\right)\right) * \cdots \\
& * \Psi^{m-1}\left(M_{b}\left(x_{1}, y_{0}, g_{2(m-n)} t\right)\right) * \Psi^{m}\left(M_{b}\left(x_{0}, y_{0}, g_{2(m-n)+1} t\right)\right) .
\end{aligned}
$$

Now as $n, m \rightarrow \infty$, we have $M_{b}\left(x_{n}, y_{m}, t\right) \rightarrow 1$ for all $t>0$. Apply same lines of the proof of Theorem 3.1 here. We have, if $u \in X \cap Y$ is a unique limit of sequences $\left\{x_{n}\right\}$ and $\left\{y_{n}\right\}$, then we have to show that $u$ is a fixed point of $T$. Since we have $M_{b}\left(x_{n}, u, t\right) \rightarrow t$ for all $t>0$ and $M_{b}\left(x_{n+1}, T(u), t\right)=M_{b}\left(T\left(x_{n}\right), T(u), t\right) \geqslant$ $\Psi\left(M_{b}\left(x_{n}, u, t\right)\right) \geqslant M_{b}\left(x_{n}, u, t\right)$, and it follows that $x_{n+1} \rightarrow T(u)$, which implies that $T(u)=u$.

Example 3.6. Let $X=\{1,3,20,31\}, Y=\{1,2\}, a * b=a b$ for all $a, b \in[0,1]$ and define

$$
M_{b}(x, y, t)=\frac{\min \{x, y\}+t}{\max \{x, y\}+t} \text { for all } x \in X, y \in Y \text { and for all } t>0 .
$$

Then $\left(X, Y, M_{b}, *\right)$ is an complete fuzzy bipolar metric space. Now, define $\Psi:(0,1] \rightarrow(0,1]$ such that $\Psi(\kappa)=\sqrt{\kappa}$. Clearly, $\Psi(\kappa)=\sqrt{\kappa}$ satisfies conditions of $\Psi$ function.

Let $\mathrm{T}: \mathrm{X} \cup \mathrm{Y} \rightarrow \mathrm{X} \cup \mathrm{Y}$ be a mapping such that $\mathrm{T}(1)=\mathrm{T}(2)=\mathrm{T}(3)=1, \mathrm{~T}(20)=\mathrm{T}(31)=3$. Then all the conditions of Theorem 3.5 are satisfied. The fixed point of $T$ is $x=1$. 
Corollary 3.7. Let $\left(\mathrm{X}, \mathrm{Y}, \mathrm{M}_{\mathrm{b}}, *\right)$ be a complete fuzzy bipolar metric space and $\mathrm{T}: \mathrm{X} \cup \mathrm{Y} \rightarrow \mathrm{X} \cup \mathrm{Y}$ a mapping satisfying:

(i) $\mathrm{T}(\mathrm{X}) \subseteq \mathrm{Y}$ and $\mathrm{T}(\mathrm{Y}) \subseteq \mathrm{X}$;

(ii) for $x \in X, y \in Y$ and $t>0, M_{b}(x, y, t)>0 \Longrightarrow M_{b}(T(y), T(x), t) \geqslant \Psi\left(M_{b}(x, y, t)\right)$.

Then $\mathrm{T}$ has a fixed point.

Proof. Proof of the Corollary follows on the lines of proof of the Theorem 3.5 and Corollary 3.3.

\section{Applications}

As a consequence to fuzzy Banach contraction result, we obtain existence of a fixed point in fuzzy bipolar metric space.

Theorem 4.1. Let $\left(\mathrm{X}, \mathrm{Y}, \mathrm{M}_{\mathrm{b}}, *\right)$ be a complete fuzzy bipolar metric space such that

$$
\lim _{t \rightarrow \infty} M_{b}(x, y, t)=1 \text { for all } x \in X, y \in Y .
$$

Let $\mathrm{T}: \mathrm{X} \cup \mathrm{Y} \rightarrow \mathrm{X} \cup \mathrm{Y}$ be mapping satisfying

(i) $\mathrm{T}(\mathrm{X}) \subseteq \mathrm{X}$ and $\mathrm{T}(\mathrm{Y}) \subseteq \mathrm{Y}$;

(ii) $\int_{0}^{M_{b}(T(x), T(y), k t)} \Upsilon(t) d t \geqslant \int_{0}^{M_{b}(x, y, t)} \Upsilon(t) d t$ for all $x \in X, y \in Y, t>0$,

where $\mathrm{k} \in(0,1)$ and $\Upsilon \in \Theta$. Then $\mathrm{T}$ has a unique fixed point.

Theorem 4.2. Let $\left(X, Y, M_{b}, *\right)$ be a complete fuzzy bipolar metric space such that $\lim _{t \rightarrow \infty} M_{b}(x, y, t)=1$ for all $x \in X, y \in Y$. Let $T: X \cup Y \rightarrow X \cup Y$ be mapping satisfying

(i) $\mathrm{T}(\mathrm{X}) \subseteq \mathrm{Y}$ and $\mathrm{T}(\mathrm{Y}) \subseteq \mathrm{X}$;

(ii) $\int_{0}^{M_{b}(T(y), T(x), k t)} \Upsilon(t) d t \geqslant \int_{0}^{M_{b}(x, y, t)} \Upsilon(t) d t$ for all $x \in X, y \in Y \quad t>0$,

where $\mathrm{k} \in(0,1)$ and $\Upsilon \in \Theta$. Then $T$ has a unique fixed point.

Proof. Proof of both Theorems 4.1 and 4.2 follows immediately from Theorem 3.1 and Corollary 3.3, respectively, by assuming $\Upsilon(t)=1$ in the above results.

\section{Acknowledgment}

The First author would like to thank the CSIR-HRDG Fund under grant EMR09/386(0059)/2017-EMR1 for financial support.

\section{References}

[1] A. George, P. Veeramani, On some results in fuzzy metric spaces, Fuzzy Sets and Systems, 64 (1994), 395-399. 1, 1.1

[2] M. Grabiec, Fixed points in fuzzy metric spaces, Fuzzy Sets and Systems, 27 (1988), 385-389. 1

[3] V. Gregori, A. Sapena, On fixed point theorems in fuzzy metric spaces, Fuzzy Sets and Systems, 125 (2002), 245-252. 1

[4] V. Gupta, N. Mani, A. Saini, Fixed point theorems and its applications in fuzzy metric spaces, Conference Paper, 2013 (2013), 11 pages. 1, 1.6

[5] G. N. V. Kishore, R. P. Agarwal, B. Srinuvasa Rao, R. V. N. Srinuvasa Rao, Caristi type cyclic contraction and common fixed point theorems in bipolar metric spaces with applications, Fixed Point Theory Appl., 2018 (2018), 13 pages. 1

[6] I. Kramosil, J. Michalek, Fuzzy metric and statistical metric spaces, Kybernetica, 11 (1975), 326-334. 1, 1

[7] D. Mihet, A Banach contraction theorem in fuzzy metric spaces, Fuzzy Sets and Systems, 144 (2004), 431-439. 3

[8] S. N. Mishra, N. Sharma, S. L. Singh, Common fixed points of maps on fuzzy metric spaces, Internat. J. Math. Math. Sci., 17 (1994), 253-258. 1.3

[9] A. Mutlu, U. Gürdal, Bipolar metric spaces and some fixed point theorems, J. Nonlinear Sci. Appl., 9 (2016), $5362-5373$. $1,1.4$ 
[10] A. Mutlu, K. Özkan, U. Gürdal, Coupled fixed point theorems on bipolar metric spaces, Eur. J. Pure Appl. Math., 10 (2017), 655-667. 1

[11] A. Mutlu, K. Özkan, U. Gürdal, Fixed point theorems for multivalued mapping on bipolar metric spaces, Fixed Point Theory, (In Press).

[12] A. Mutlu, K. Özkan, U. Gürdal, Locally and weakly contractive principle in bipolar metric spaces, TWMS J. App. Eng. Math., (In Press). 1

[13] B. Schweizer, A. Sklar, Statistical metric spaces, Pacific J. Math., 10 (1960), 313-334. 1

[14] L. A. Zadeh, Fuzzy sets, Inf. Control, 8 (1965), 338-353. 1 